\title{
Anakinra treatment for systemic juvenile idiopathic arthritis and adult onset Still disease
}

\section{Patricia Woo}

Systemic juvenile idiopathic arthritis (sJIA) is a distinct clinical spectrum of illnesses compared to other types of chronic arthritides in children. Typically, the clinical features include the characteristic quotidian fevers, evanescent rashes, serositis, lymphadenopathy and hepatosplenomegaly in addition to persistent and destructive arthritis at the severe end of the spectrum. The children also appear to be more prone to macrophage activation syndrome/secondary histiolymphocytic haemophagocytosis (HLH). It is generally acknowledged that a similar disease occurs rarely in adults: adult onset Still disease (AOSD). However, the clinical classifications for each were developed separately and are different from each other. The Yamaguchi AOSD criteria ${ }^{1}$ are less precise in the exclusions and are able to include a wider spectrum of clinical manifestations as compared to the International League of Associations for Rheumatology (ILAR) classification of sJIA. ${ }^{2}$

The pathogenesis of sJIA is still the subject of much research and the first reports of the role of interleukin (IL)- 1 in sJIA by Verbsky et $a l^{3}$ and Pascual et $a l^{4}$ have raised the hope that both the pathogenesis and treatment of this disease are finally being elucidated. Similarly in AOSD, Fujii et $a l^{5}$ and Fitzgerald et $a l^{6}$ found success in treating AOSD with anakinra, a recombinant form of the IL-1 receptor antagonist. The paper by Lequerre et $a l^{7}$ in this issue (see page 302) supports the anecdotal reports at scientific meetings of anakinra treatment failures in sJIA, as well as the dramatic benefit anakinra produced in responders.

In this report, the authors performed a prospective study among paediatric rheumatologolists and rheumatologists to assess the efficacy and safety of anakinra given to 20 sJIA and 15 AOSD patients. The response criteria are standard ones

Correspondence to: Professor Patricia Woo, University College London, Paediatric and Adolescent

Rheumatology Department of Immunology and Molecular Pathology 46, Cleveland Street London, W1T 4JF UK; patricia.woo@ucl.ac.uk now used for all therapeutic trials, but they are different for the two groups: ACR pedi 30 (American College of Rheumatology 30\% improvement (pediatric) of a composite score) for JIA and ACR 20 (20\% improvement in an adult orientated composite score) for the adults. Their results are interesting in that there appears to be a dichotomy between the two groups in terms of the percentage of complete responders (4/20 sJIA vs $9 / 15$ AOSD), and non-responders (10/20 sJIA vs $2 / 15 \mathrm{AOSD})$. Complete response is dramatic in both groups. However, a further five sJIA patients had a partial response and steroid dose was tapered. The anakinra dosages for the sJIA patients were not the same (1-2 $\mathrm{mg} / \mathrm{kg}$ variation), and it could be argued that this response rate is due to the pharmcodynamics not being optimal in some of these children. The concomitant disease-modifying antirheumatic drug (DMARD) used also may affect the general outcome as the authors suggested in the discussion. However, these factors may not be the whole answer in that body size and DMARD use are not particularly different in the complete response cohort vs the nonresponder cohort. However, the numbers are small in this sort of analysis of subgroups, and any conclusions can only be undertaken with caution. In two sJIA patients another phenomenon was seen: the decrease of efficacy with time, whereas this was not seen in the AOSD cohort.

So what have we learnt from this paper? The first issue is whether there is a real difference in phenotype between AOSD and sJIA, and so affecting the response rate to anakinra. Although the spectra of clinical symptoms are similar, there is the clear difference of the age of onset, which may be related more to genetic factors. This paper suggests that there may be a difference in the response rate to anakinra between the two groups but the classification criteria as well as the measurement of outcome are similar but not the same. So these variations may be artificial. Harmonising of classification and outcome criteria would be useful in future in comparisons of this sort so that differences in response rate would be more informative in terms of pathology as well as the efficacy of the drug.

Despite the small numbers in each group, there are reports of infections, which may or may not be directly due to anakinra. Since this drug is used off licence in many units where conventional therapies have failed, there should be close monitoring during treatment. A recent report of "severe systemic inflammatory response syndrome" to anakinra in an AOSD patient ${ }^{8}$ also raises the possibility of this adverse event in children. For both groups of patients, phase II and III studies are needed to assess efficacy and safety, which unfortunately is not likely to be carried out in the case of anakinra. The rheumatology community should ensure that these are carried out for newer biologics that block IL-1 signalling.

There is much evidence clinically as well as genetically that sJIA represent a spectrum of illnesses from mild to very severe. ${ }^{9}$ The variable response to the use of IL-1 signal blockade with anakinra supports the notion that there is a clinical as well as a pathological spectrum. The blockade of IL-1 signalling has a dramatic and sustained effect in some patients (the complete responders), with cessation of symptoms and significant decrease of acute phase markers. The large group of partial responders and non-responders are suggestive of pathological processes that are independent of the IL-1 pathway, rather than just a difference in pharmacodynamics and pharmacokinetics. These response rates are in a way reminiscent of the use of anti-tumour necrosis factor (TNF) in sJIA, reported by one of the authors of the paper under discussion in this issue. ${ }^{10}$ Blockade of IL- 6 signalling however, has been reported in phase II studies with much better response rates. ${ }^{11}{ }^{12}$ This response rate is likely to be due to the fact that IL- 6 is able to be stimulated by IL-1 and TNF, and so blockade of IL- 6 will take care of processes that come mainly from the IL-1 or TNF pathways, as well other sources of stimulation of IL- 6 in this disease. Much has already been written about the importance of IL- 6 in the pathogenesis of sIIA. ${ }^{13} 14$ In order to achieve complete response in most if not all of the patient with therapy, a different approach to current drug trial design is necessary. Not only statistical power should be achieved by a multi-centre study, which is multi-ethnic, but also account must be taken of genetic factors, which inevitably 
have a part to play in the clinical phenotypes as well as drug response. Thus, comprehensive whole genome variation analysis is a very important way forward to characterise the pathogenesis as well as drug response and should be performed in parallel with gene expression/protein profiling in future biological drug trials in sJIA.

Competing interests: None declared.

Accepted 3 January 2008

Ann Rheum Dis 2008:67:281-282.

doi:10.1136/ard.2007.082859

\section{REFERENCES}

1. Yamaguchi M, Ohta A, Tsunematsu T, Kasukawa R, Mizushima Y, Kashiwagi H, et al. Preliminary criteria for classification of adult Still's disease. J Rheumatol 1992:19:424-30.

2. Petty RE, Southwood TR, Manners P, Baum J, Glass DN, Goldenberg J, et al. International League of Associations for Rheumatology. International League of Associations for Rheumatology classification of juvenile idiopathic arthritis: second revision, Edmonton, 2001. J Rheumatol 2004;31:390-2.

3. Verbsky JW, White AJ. Effective use of the recombinant interleukin 1 receptor antagonist anakinra in therapy resistant systemic onset juvenile rheumatoid arthritis. J Rheumatol 2004;31:2071-5.

4. Pascual V, Allantaz F, Arce E, Punaro M, Banchereau J. Role of interleukin-1 (IL-1) in the pathogenesis of systemic onset juvenile idiopathic arthritis and clinical response to IL-1 blockade. J Exp Med 2005:201:1479-86.

5. Fujii T, Nojima T, Yasuoka H, Satoh S, Nakamura K, Kuwana $\mathrm{M}$, et al. Cytokine and immunogenetic profiles in Japanese patients with adult-onset Still's disease. Association with chronic articular disease. Rheumatology 2001;40:1398-404.

6. Fitzgerald AA, Leclercq SA, Yan A, Homik JE, Dinarello CA. Rapid responses to anakinra in patients with refractory adult-onset Still's disease. Arthritis Rheum 2005:52:1794-803.

7. Lequerré $T$, Quartier $P$, Rosellini $D$, Alaoui $F$ De Bandt M, Mejjad O, Kone-Paut I, et al. Interleukin-1 receptor aganist (anakinra) treatment in patients with systemic-onset juvenik idiopathic arthritis or adult-onset Still disease: preliminary experience in France. Ann Rheum Dis 2008;67: 302-8.
8. Guignard S, Dien G, Dougados M. Severe systemic inflammatory response syndrome in a patient with adult onset Still's disease treated with the anti-IL1 drug anakinra: a case report. Clin Exp Rheumatol 2007;25:758-9

9. Woo P. Systemic juvenile idiopathic arthritis. Nat Clin Pract Rheumatol 2006:2:28-34.

10. Quartier $\mathbf{P}$, Taupin $\mathrm{P}$, Bourdeaut $F$, Lemelle I, Pillet $\mathrm{P}$ Bost $\mathrm{M}$, et al. Efficacy of etanercept for the treatment of juvenile idiopathic arthritis according to the onset type. Arthritis Rheum 2003:48:1093-101.

11. Yokota S, Miyamae T, Imagawa T, Iwata N, Katakura S, Mori M, et al. Therapeutic efficacy of humanized recombinant anti-interleukin-6 receptor antibody in children with systemic-onset juvenile idiopathic arthritis. Arthritis Rheum 2005;52:818-25.

12. Woo P, Wilkinson N, Prieur AM, Southwood T, Leone V, Livermore $\mathrm{P}$, et al. Open label phase II trial of single, ascending doses of MRA in Caucasian children with severe systemic juvenile idiopathic arthritis: proof of principle of the efficacy of IL-6 receptor blockade in this type of arthritis and demonstration of prolonged clinical improvement. Arthritis Res Ther 2005;7:1281-8.

13. Woo $\mathbf{P}$. The cytokine network in juvenile chronic arthritis. Rheum Dis Clin North Am 1997;23:491-8.

14. De Benedetti F, Martini A. Is systemic juvenile rheumatoid arthritis an interleukin 6 mediated disease? J Rheumatol 1998;25:203-7

\title{
Date for your diary
}

\author{
International Forum on Quality \& Safety in Health Care \\ 22-25 April 2008 \\ Le Palais de Congrès de Paris \\ Paris, France \\ http://www.internationalforum.bmj.com \\ Why attend? \\ - Join over 1000 healthcare professionals from over 40 countries worldwide \\ - Learn from experienced leaders and improvement experts \\ - Find out what is current in quality and safety \\ - Gain new skills and tools for creating change in your organisation \\ - Take home practical solutions for improvement methods \\ - Network with like-minded colleagues
}

This is a premier event for those dedicated to improving quality and safety in healthcare. Our focus is on practical, take-home ideas that will leave you inspired to implement valuable, lasting change on your return to the workplace.

Register online from January 2008 onwards

For more information on the forum visit - http://www.internationalforum.bmj.com 